J. Clin. Chem. Clin. Biochem.

Vol. 14, 1976, pp. 581-587

\title{
Aktivierung der sauren Prostataphosphatase durch 1-Pentanol
}

\section{Von H. Gallati}

\section{Diagnostische Forschungsabteilung der F. Hoffmann-La Roche \& Co. AG Basel}

und M. Roth

Laboratoire Central, Hôpital Cantonal, Genève

(Eingegangen am 19. August/8. Oktober 1976)

Zusammenfassung: Die Aktivität der sauren Prostataphosphatase wird durch Zusatz von $150 \mathrm{mmol} / \mathrm{l}$ 1-Pentanol zum Testansatz um $90 \%$ erhöht. Diese Aktivierung zeigt sich in einem vermehrten Substratumsatz, indem der Phosphomonoester schneller gespalten wird und dadurch eine entsprechend größere Menge des freigesetzten, organischen Rests nachgewiesen werden kann. Die Menge des freien Phosphats entspricht hingegen nicht dem Substratumsatz, da durch eine Transphosphorylierungs-Reaktion ein Teil des Phosphatrests vom Substrat auf das 1-Pentanol übertragen wird.

In der vorliegenden Arbeit wird der Einfluß des Substrats, des Puffers, des pH und des $L$-Tartrats auf die 1-Pentanolaktivierte Prostataphosphatase untersucht.

\section{Activation of acid prostate phosphatase by 1-pentanol}

Summary: The activity of the acid phosphatase from prostate was increased by $90 \%$ by the addition of $150 \mathrm{mmol} / \mathrm{l}$ 1-pentanol to the assay mixture. This activation results in an increased turnover of substrate, so that the phosphomonoester is cleaved more rapidly and a correspondingly larger amount of the released organic residue can be detected. The quantity of free phosphate, however, does not correspond to the substrate turnover, because some of the phosphate residue is transferred from the substrate to the 1-pentanol in a transphosphorylation reaction. The influence of the substrate, buffer, $\mathrm{pH}$ and of tartrate on the 1-pentanol-activated prostate phosphatase was investigated.

\section{Einführung}

Alkalische Phosphatasen verschiedenen Ursprungs werden durch Aminoalkohole aktiviert, indem durch eine zusätzliche Transphosphorylierungsiéaktion ein Teil des Phosphatrests vom Substrat auf den Aminoalkohol übertragen wird (1-9). Die Deutsche Gesellsçhaft für Klinische Chemie empfiehlt daher für die Standardmethode zur Bestimmung der alkalischen Phosphatase im Serum oder Plasma der Testlösung $1 \mathrm{~mol} / \mathrm{l}$ Diäthananolamin beizufügen (10).

Im Bestreben, auch für die diagnostisch wichtige Prostataphosphatase (EC 3.1.3.2) einen Aktivator zu finden, wurden verschiedene Substanzklassen untersucht und dabei überraschend festgestellt, daß längerkettige Alko- hole den Substratumsatz der Prostataphosphatase wesentlich zu steigern vermögen.

In dieser Arbeit wird der Einfluß verschiedener Alkohole auf die Aktivität der sauren Prostataphosphatase untersucht und die Testbedingungen zur Bestimmung der 1Pentanol-aktivierten Prostataphosphatase bezüglich Substrat, Puffer, pH und Tartratkonzentration optimiert.

\section{Material und Methoden}

Die saure Prostataphosphatase wurde mit $20 \mathrm{mmol} / 1$ Natriumacetatpuffer $\mathrm{pH}$ 5,5 aus menschlichem Prostatagewe be extrahiert, der pH-Wert mit $100 \mathrm{mmol} / 1$ Essigsäure auf 4,5 erniedrigt und das Fremdprotein mit $5000 \mathrm{~g} 15 \mathrm{~min}$. abzentrifugiert. Die Lösung wurde nun auf Carboxymethyl-Cellulose mit Natriumacetatpuffer $20 \mathrm{mmol} / \mathrm{l} \mathrm{pH} \mathrm{4,5}$ und einem linearen Natrium- 
chlorid-Gradienten von 0-500 mmol/l chromatographiert. Die gepoolten aktiven Fraktionen wurden eingeengt und auf Sephadex G-150 mit Natriumacetatpuffer $0,1 \mathrm{~mol} / 1 \mathrm{pH} \mathrm{5,5}$ weiter gereinigt.

Die spezifische Aktivität dieses Prostataphosphatase-Präparates betrug $500 \mathrm{U} / \mathrm{mg}$ Protein (nach 1. c. (11)).

Die verwendeten Chemikalien waren analysenrein. Die Alkohole wurden von Merck (Darmstadt), die Substrate 4-Nitrophenylphosphat, Phenylphosphat, 2-Glycerophosphat und Adenosin5-monophosphat von Fluka, CM-Cellulose von Whatmann und Sephadex G-150 von Pharmacia bezogen.

Zur Aktivitätsbestimmung der sauren Prostataphosphatase wurde $0,1 \mathrm{ml}$ Enzymlösung zu $0,5 \mathrm{ml}$ vorgewärmter Substratpufferlösung zugemischt und nach 30 Minuten Inkubation bei $37^{\circ} \mathrm{C}$ die Aktivität nach Bessey et al. ((11), 4-Nitrophenylphosphat), Bastiaanse ((12), 2-Glycerophosphat, Adenosin-5'-monophosphat) oder Folin ((13), Phenylphosphat) bestimmt.

\section{Resultate}

\section{Einfluß verschiedener Alkohole auf die Aktivität} der sauren Prostataphosphatase

Zur Aktivitätsbestimmung der Prostataphosphatase wurden dem Testansatz $(0,1 \mathrm{~mol} / 1$ Natriumcitrat, $\mathrm{pH} 5,25$ und $5 \mathrm{mmol} / 1$ 4-Nitrophenylphosphat) unterschiedliche Mengen der in Abbildung 1 aufgeführten Alkohole zugefügt und die entsprechenden Resultate in Prozent des alkoholfreien Testansatzes angegeben. Die Aktivierung

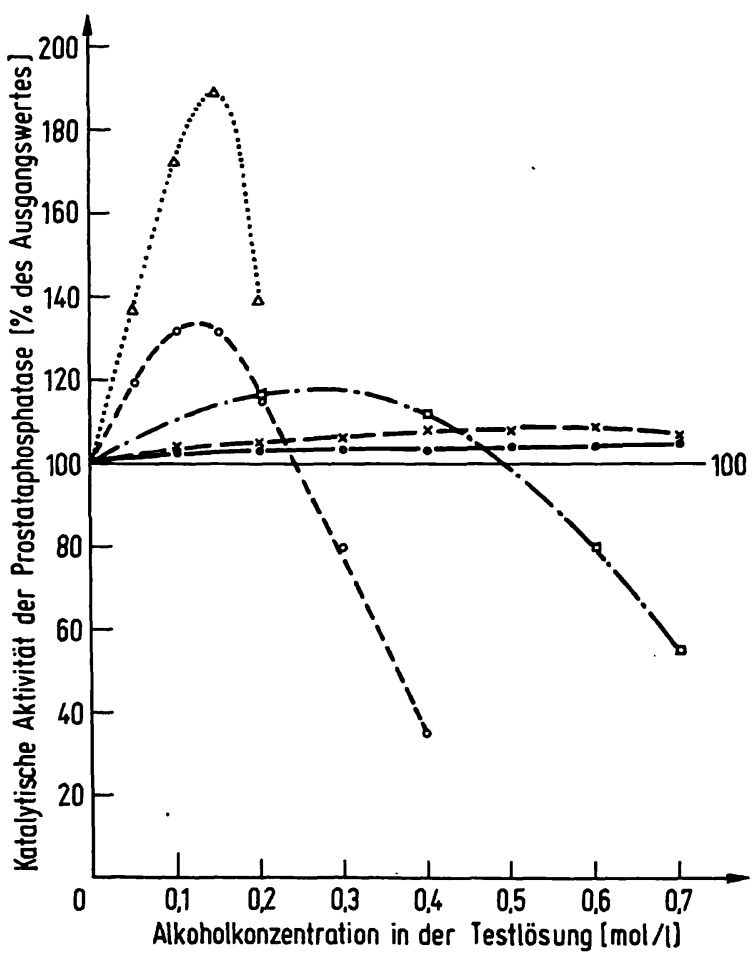

Abb. 1. Einfluß verschiedener Alkohole auf die Aktivität der Prostataphosphatase.

Aktivitätsbestimmung der Prostataphosphatase in 0,1 $\mathrm{mol} / \mathrm{l}$ Citratpuffer $\mathrm{pH}$ 5,25 mit $5 \mathrm{mmol} / \mathrm{l}$ 4-Nitrophenylphosphat und verschiedenen Konzentrationen an Methano

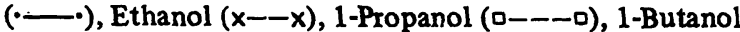
$(0--\infty)$ und 1-Pentanol $(\Delta \cdots . . \Delta)$. der Prostataphosphatase beträgt mit $150 \mathrm{mmol} / 1$ 1-Pentanol 90\%, mit $150 \mathrm{mmol} / 1$ 1-Butanol 31\%, mit 300 $\mathrm{mmol} / \mathrm{l}$ 1-Propanol $18 \%$ und mit $600 \mathrm{mmol} / \mathrm{l}$ Ethanol 9\%. Anderseits üben Methanol und Ethanol bis zu einer Konzentration von $1 \mathrm{~mol} / \mathrm{l}$ keinen hemmenden Einfluß auf die Prostataphosphatase aus, während 1-Propanol von $500 \mathrm{mmol} / 1$ und 1 -Butanol von $250 \mathrm{mmol} / 1$ an die Enzymaktivität vermindern.

Mit $100 \mathrm{mmol} / \mathrm{l} 1$-Hexanol wird die Prostataphosphatase um $44 \%$ und mit $50 \mathrm{mmol} / 1$ 1-Heptanol um $22 \%$ aktiviert. Wegen der schlechten Löslichkeit kann die Konzentration dieser beiden Alkohole nicht weiter gesteigert werden.

2-Pentanol (150 mmol/1) aktiviert die Prostataphosphatase um $80 \%$, 3-Pentanol $(150 \mathrm{mmol} / \mathrm{l})$ um $25 \%$ und 1,5 -Pentandiol $(200 \mathrm{mmol} / \mathrm{l})$ um $50 \%$. Durch $D$-Sorbit wird die Aktivität der Prostataphosphatase nicht beeinflußt.

Aminoalkohole, Aminosäuren, Ketosäuren, Hydroxysäuren, Mono-, Di- und Tricarbonșäuren haben auf die Prostataphosphatase-Aktivität keinen oder dann nur einen hemmenden Einfluß (Tab. 1).

Einfluß der Art und der Konzentration des Sub. strates auf die 1-Pentanol-aktivierte Prostataphosphatase

Die Michaeliskonstante der Prostataphosphatase für das 4-Nitrophenylphosphat wurde in Abhängigkeit zur 1-Pentanol-Konzentration bestimmt und die entsprechen-

Tab. 1. Einfluß verschiedener Substanzen auf die Aktivität der Prostataphosphatase.

Aktivitätsbestimmung der Prostataphosphatase in 0,1 mol/1 Natriumacetat pH 5,25 mit 5 mmol/l 4-Nitrophenylphosphat und mit folgenden Zusätzen in $\mathrm{Ab}$ wesenheit von 1-Pentanol.

\begin{tabular}{lc}
\hline Zusatz & $\begin{array}{c}\text { Prostataphosphatase- } \\
\text { Aktivität (in \%) }\end{array}$ \\
\hline Ohne Zusatz & 100 \\
$500 \mathrm{mmol} / 1$ 2-Ethylaminoethanol & 66 \\
$500 \mathrm{mmol} / 1$ Triethanolamin & 53 \\
$500 \mathrm{mmol} / 1$ Diethanolamin & 43 \\
$500 \mathrm{mmol} / 1$ Ethanolamin & 34 \\
$50 \mathrm{mmol} / 1$ L-Alanin & 100 \\
$50 \mathrm{mmol} / 1$ L-Glutaminsäure & 100 \\
$50 \mathrm{mmol} / 1$-Asparaginnäure & 93 \\
$50 \mathrm{mmol} / 1$ L-Cystein & 92 \\
$50 \mathrm{mmol} / 1$ 8-Aminocaprylsäure & 54 \\
$50 \mathrm{mmol} / 1$ Brenztraubensäure & 72 \\
$50 \mathrm{mmol} / 1$ 2-Oxobuttersäure & 93 \\
$50 \mathrm{mmol} / 1$-Lactat & 86 \\
$50 \mathrm{mmol} / 1$ 2-Hydroxybuttersäure & 80 \\
$50 \mathrm{mmol} / 1$ Oxalsäure & 54 \\
$50 \mathrm{mmol} / 1$ Bernsteinsäure & 100 \\
$50 \mathrm{mmol} / 1$ 2-Oxoglutarsäure & 100 \\
$50 \mathrm{mmol} / 1$ DL-Äpfelsäure & 72 \\
$50 \mathrm{mmol} / 1$ D-Äpfelsäure & 59 \\
$50 \mathrm{mmol} / 1$ L-Äpfelsäure & 91 \\
$50 \mathrm{mmol} / 1$ D-Tartrat & 100 \\
$50 \mathrm{mmol} / 1$ L-Tartrat & 0 \\
\hline
\end{tabular}




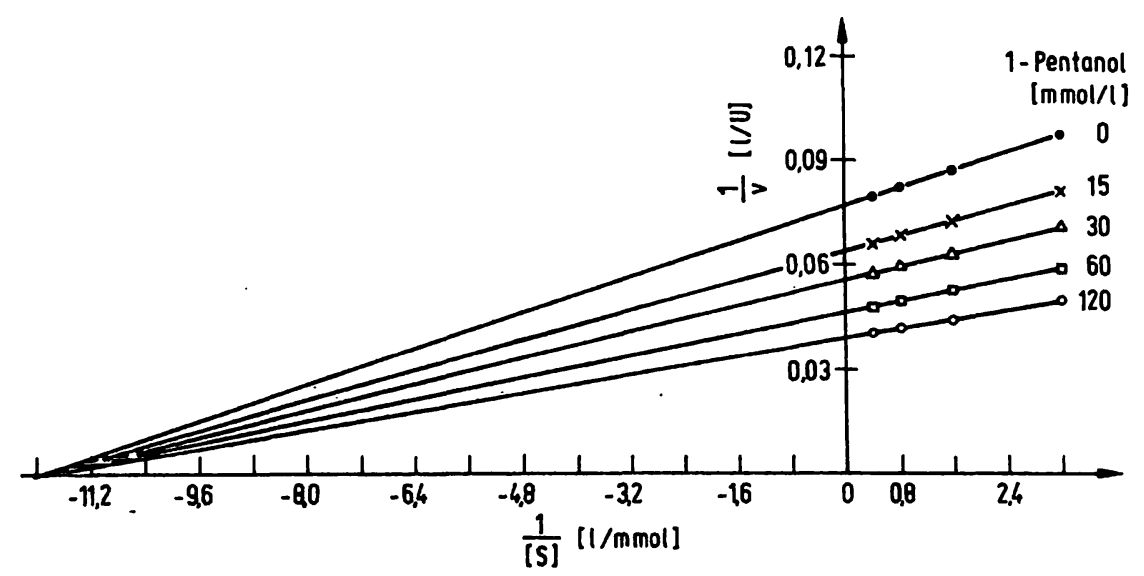

Abb. 2. Michaeliskonstante der Prostataphosphatase für 4-Nitrophenylphosphat in Abhängigkeit der 1-Pentanol-Konzentration. Aktivitätsbestimmung der Prostataphosphatase in 0,1 mol/1 Natriumacetatpuffer pH 5,25 mit verschiedenen Konzentrationen an 1-Pentanol und 4-Nitrophenylphosphat. Darstellung nach Lineweaver-Burk.

den Resultate nach der Darstellungsweise von LineweaverBurk in Abbildung 2 aufgezeichnet. Daraus ist ersichtlich, daß die Substrataffinität durch den Zusatz verschiedener Mengen von 1-Pentanol zum Testansatz nicht beeinflußt wird. Die Michaeliskonstante für 4-Nitrophenylphosphat beträgt unter den gegebenen Testbedingungen $(0,1 \mathrm{~mol} / 1$. Natriumacetat vom pH 5,25) bei allen 1-Pentanol-Konzentrationen $83,3 \mu \mathrm{mol} / 1$. Hingegen nimmt die maximal mögliche Reaktionsgeschwindigkeit (V) mit steigender Konzentration an 1-Pentanol deutlich zu.

1-Pentanol verhält sich der Prostataphosphatase gegenüber ähnlich wie ein Substrat. So beträgt unter den gegebenen Testbedingungen $(0,1 \mathrm{~mol} / 1$ Natriumacetat, pH 5,25) die Michaeliskonstante für das 1-Pentanol 250 $\mathrm{mmol} / \mathrm{l}$. Die Affinität des 1-Pentanols zur Prostataphosphatase wird durch die unterschiedliche Substratkonzentration nicht beeinflußt.

Mit Phenylphosphat als Substrat zur Bestimmung der Prostataphosphatase tritt bei Zusatz von $150 \mathrm{mmol} / 1$ 1-Pentanol zum Testansatz ebenfalls eine Aktivierung von $90 \%$ auf. Die Michaeliskonstante für Phenylphosphat wird durch 1-Pentanol nicht beeinflußt. Sie beträgt unter den gegebenen Testbedingungen $(0,1 \mathrm{~mol} / 1$ Natriumcitrat vom $\mathrm{pH} \mathrm{5,25)} 200 \mu \mathrm{mol} / 1$.

Völlig anders verhält es sich, wenn als Substrat 2-Glycerophosphat oder Adenosin- $5^{\prime}$-monophosphat eingesetzt und die Aktivität der Prostataphosphatase durch Messung der freigesetžtèn Phosphationen bestimmt wird. Statt einer Aktivierung tritt bei Anwesenheit von 1-Pentanol scheinbar eine Aktivitätshemmung auf, die mit steigendem Alkoholgehalt zunnimmt und bei einer Konzentration von $150 \mathrm{mmol} / 1$ 1-Pentanol 40\% erreicht (Abb. 3). Dabei wird auch für diese beiden Substrate die Michaeliskon: stante durch Zusatz von 1-Pentanol zur Testlösung nicht verändert. Unter den gegebenen Testbedingungen $(0,1$ mol/1 Natriumcitrat, pH 5,25) beträgt die Michaelis: konstante fự Adenosin-5'-monophosphat $5,26 \mathrm{mmol} / 1$ und für 2-Glycerophosphat $4,0 \mathrm{mmol} / \mathrm{l}$.

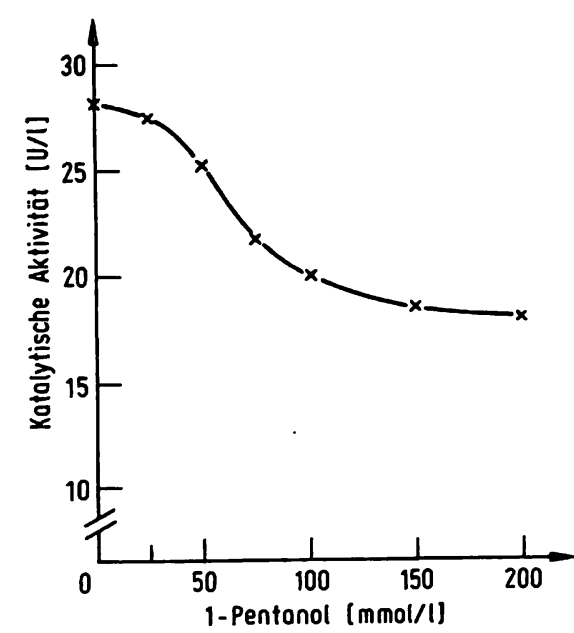

Abb. 3. Einfluß von 1-Pentanol auf die Aktivitätsmessung der Prostataphosphatase mit 2-Glycerophosphat.

Aktivitätsmessung der Prostataphosphatase in $0,1 \mathrm{~mol} / 1$ Natriumcitratpuffer pH 5,25 mit $25 \mathrm{mmol} / 1$ 2-Glycerophosphat und mit verschiedenen Konzentrationen an 1-Pentanol (nach l. c. (12)).

Auf Grund dieses widersprïchlichen Verhaltens des 1-Pentanols gegenüber der Prostataphosphatase wurde in einem weiteren Versuch zur Aktivitätsmessung die Konzentration sowohl des abgespaltenen, organischen Rests wie auch der freigesetzten Phosphationen bestimmt. Mit 3,0 mmol/1 4-Nitrophenylphosphatlösung in $0,1 \mathrm{~mol} / 1$ Natriumcitrat pH 5,25 wurde Prostataphosphatase bei $37^{\circ} \mathrm{C}$ inkubiert und nach bestimmten Zeitintervallen in einem Teil der Inkubationslösung gleichzeitig der Gehalt an 4-Nitrophenol und an organischem Phosphat bestimmt. Im alkoholfreien Testansatz waren die beiden Spaltprodukte bei jeder Messung in gleichmolarer Konzentration vorhanden und erreichten nach Erschöpfung des Substrates die Konzentration von $3 \mathrm{mmol} / 1$. Eine Zugabe von $150 \mathrm{mmol} / \mathrm{l} 1$-Pentanol zu der obigen Testlösung bewirkte, daß während der enzymatischen Reaktion doppelt soviel 4-Nitrophenol gefunden wurde wie anorganisches Phosphat, und daß 


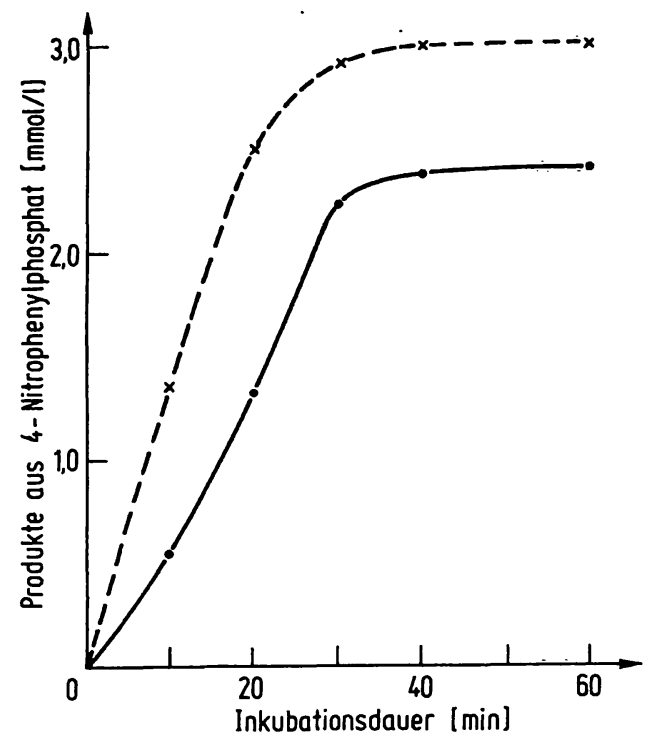

Abb. 4. Messung von 4-Nitrophenol $(x---x)$ und anorganischem Phosphat $(\cdot-)$ nach $0-60 \mathrm{~min}$.

Reaktion der Prostataphosphatase mit 4-Nitrophenylphosphat $3 \mathrm{mmol} / 1$ in Natriumcitrat $100 \mathrm{mmol} / \mathrm{l} \mathrm{pH} \mathrm{5,25}$ und 1 -Pentanol $150 \mathrm{mmol} / \mathrm{l}$.

nach Erschöpfung des Substrates den $3 \mathrm{mmol} / \mathrm{l}$ 4-Nitrophenol nur 2,4 mmol/1 Phosphationen gegenüberstanden (Abb. 4). Im weiteren Reaktionsverlauf nahm die Phosphatkonzentration langsam zu und erreichte nach einigen Stunden den erwarteten Wert von $3 \mathrm{mmol} / \mathrm{l}$.

Einfluß des pH-Wertes und des Puffers auf die 1-Pentanol-aktivierte Prostataphosphatase

Die pH-Aktivitätskurve der sauren Prostataphosphatase verläuft ohne Zusatz von 1-Pentanol im $\mathrm{pH}$-Bereich von 4-6 relativ flach (Abb. 5). Durch Zusatz von $150 \mathrm{mmol} / \mathrm{I}$ 1-Pentanol zum Testansatz wird die pH-Aktivitätskurve wesentlich steiler, wobei der aktivierende Einfluß des 1-Pentanols vom $\mathrm{pH} 4$ bis 5,25 stark zunimmt, um dann mit höheren pH-Werten wieder abzunehmen. Die Aktivierung der Prostataphosphatase durch 1-Pentanol ist also stark pH-abhängig, das pH-Optimum ist bei 5,25.

Das pH-Optimum wird durch unterschiedliche Konzentrationen an 4-Nitrophenylphosphat oder an 1-Pentanol nicht verändert, es ist hingegen von Substrat zu Substrat etwas verschieden.

Die maximal mögliche Reaktionsgeschwindigkeit (V) der Prostataphosphatase ist in Citrat- und Acetatpuffer identisch und sie wird auch durch eine unterschiedliche Pufferkonzentration nicht beeinflußt. Hingegen ist die Michaeliskonstante der Prostataphosphatase für das 4-Nitrophenylphosphat sowohl von der Pufferart wie auch von der Pufferkonzentration abhängig (Abb. 6 und Tab. 2). Mit zunehmender Puffer- und auch Salzkonzentration wird die Affinität des Substrats zum Enzym vermindert, und

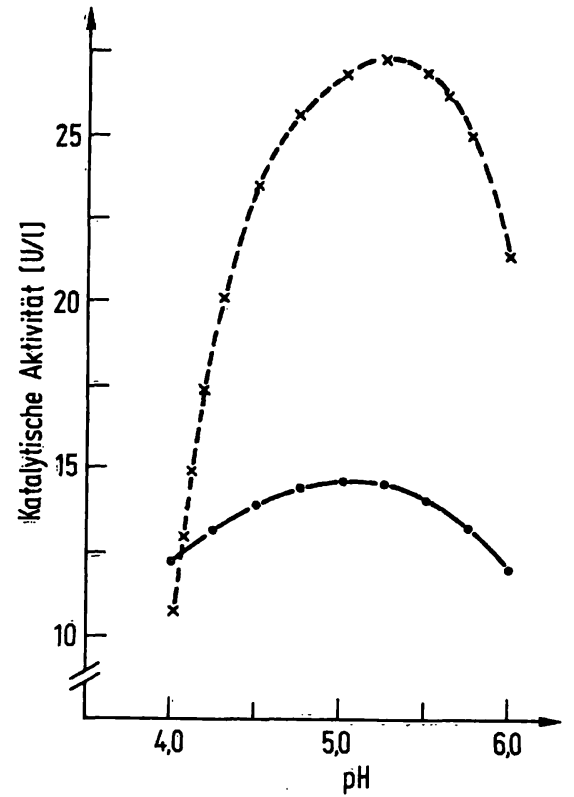

Abb. 5. pH-Aktivitätskurve der Prostataphosphatase mit und ohne 1-Pentanol-Zusatz.

Aktivitätsbestimmung der Prostataphosphatase in 0,1 mol/l Natriumcitratpuffer mit $5 \mathrm{mmol} / \mathrm{l}$ 4-Nitrophenylphosphat.

$(\cdot-\cdot)$ ohne Zusatz von 1-Pentanol und

(x---x) mit $150 \mathrm{mmol} / \mathrm{l} 1$-Pentanol.

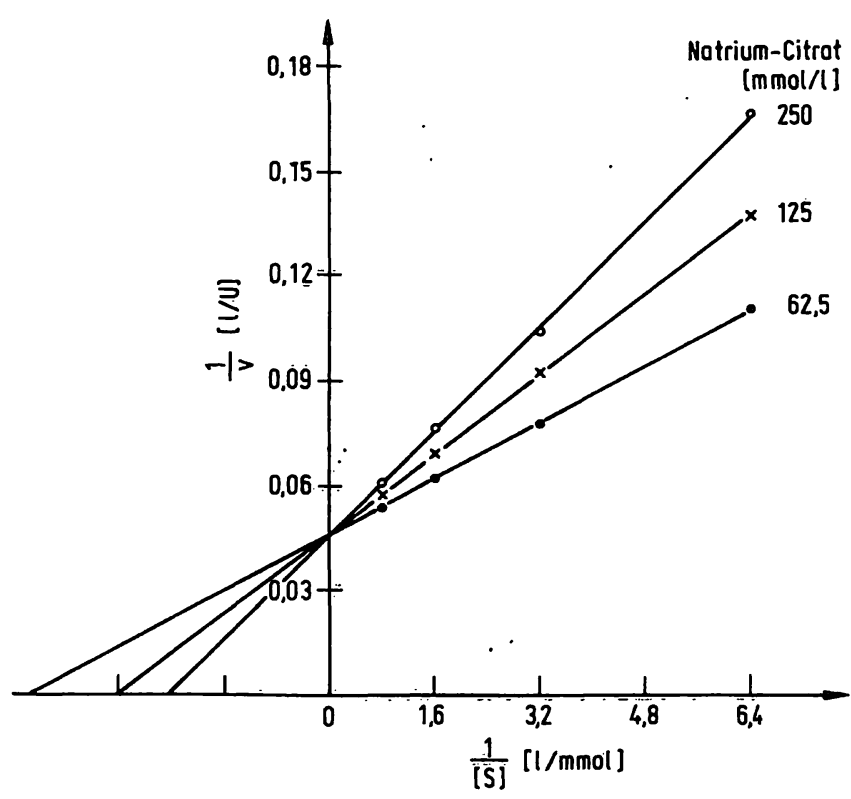

Abb. 6. Michaeliskonstante der Prostataphosphatase für 4-Nitrophenylphosphat in Abhängigkeit zur Natriumcitratkonzentration.

Aḱtivitätsbestimmung der Prostataphosphatase mit uñterschiedlicher Konzentration an 4-Nitrophenylphosphat und Natriumcitrat beim pH 5.25. Darstellung nach LineweaverBurk.

zudem ist die hạlbe Substratsättigung der Prostataphosphatase in Acetatpuffer schneller erreicht als in Citratpuffer. 
Tab. 2. Michaeliskonstante der Prostataphosphatase für 4-Nitrophenylphosphat in Abhängigkeit der Pufferart und der Puffer- und Salzkonzentration beim pH 5,25.

\begin{tabular}{|c|c|c|c|c|}
\hline \multirow[b]{2}{*}{ Puffersystem } & \multicolumn{4}{|c|}{$\begin{array}{l}\text { Michaeliskonstante }(\mu \mathrm{mol} / 1) \text { bei } \\
\text { folgenden Konzentrationen }\end{array}$} \\
\hline & $\begin{array}{l}62,5 \\
\mathrm{mmol} / \mathrm{l}\end{array}$ & $\begin{array}{l}125 \\
\mathrm{mmol} / \mathrm{l}\end{array}$ & $\begin{array}{l}250 \\
\mathrm{mmol} / \mathrm{l}\end{array}$ & $\begin{array}{l}500 \\
\mathrm{mmol} / 1\end{array}$ \\
\hline $\begin{array}{l}\text { Natriumcitrat } \\
\text { Natriumacetat } \\
\text { Natriumchlorid } \\
\text { (in } 0,1 \mathrm{~mol} / 1 \text { Acetat- } \\
\text { puffer) }\end{array}$ & $\begin{array}{r}221 \\
75 \\
-\quad\end{array}$ & $\begin{array}{l}313 \\
105 \\
230\end{array}$ & $\begin{array}{l}417 \\
160 \\
318\end{array}$ & $\begin{array}{l}- \\
\overline{496}\end{array}$ \\
\hline
\end{tabular}

Einfluß gewisser Anionen auf die 1-Pentanolaktivierte Prostataphosphatase

Die Hemmwirkung des $L$-Tartrates auf die Prostataphosphatase wird durch den Zusatz von $150 \mathrm{mmol} / 1$ 1-Pentanol deutlich verringert (Abb. 7). Zur vollständigen Hemmung der Prostataphosphatase muß daher die Tartratkonzentration auf $25 \mathrm{mmol} / \mathrm{l}$ heraufgesetzt werden.

Ethylendiamin-tetraessigsäure (EDTA) bis zu einer Konzentration von $50 \mathrm{mmol} / \mathrm{l}$ hat auf die Aktivität der Pro-

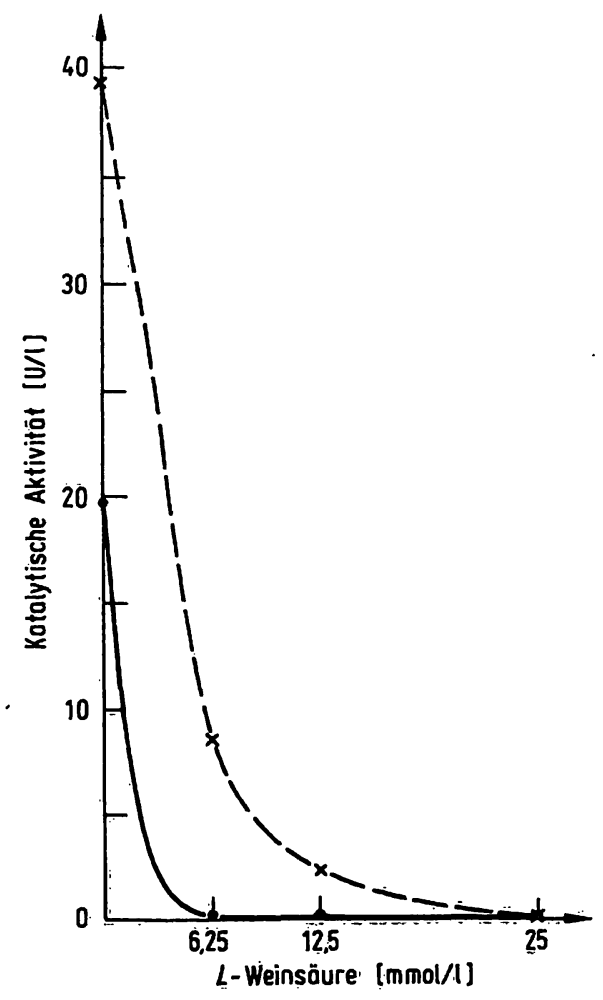

Abb. 7. Einfluß des $L$-Tartrates auf die Aktivität der Prostataphosphatase.

Aktivitätsmessung der Prostataphosphatase in $0,1 \mathrm{~mol} / 1$ Natriumcitratpuffer $\mathrm{pH}$ 5,25 mit $5 \cdot \mathrm{mmol} / \mathrm{l}$ 4-Nitrophenylphosphat und entsprechenden Meingen an $L=$ Tartrat.

(--) ohne 1-Pentanol-Zusatz resp.

(x-D-x) mit $150 \mathrm{mmol} / 1$ 1-Pentanol. stataphosphatase keinen Einfluß. Während Chlorid, Bromid und Jodid bis zu einer Konzentration von 100 $\mathrm{mmol} / 1$ die Prostataphosphatase nicht beeinflussen, vermag Fluorid schon in einer Konzentration von $2 \mathrm{mmol} / \mathrm{l}$ die Aktivität dieses Enzyms.vollständig zu hemmen. Diese Fluoridhemmung der Prostataphosphatase wird durch Zusatz von $150 \mathrm{mmol} / 1$ 1-Pentanol zum Testansatz nur unwesentlich verringert.

Einfluß der Inkubationstemperatur und der Reaktionsdauer auf die 1-Pentanol-aktivierte Prostataphosphatase

Die Spontanhiydrolyse des 4-Nitrophenylphosphates wie auch die Inaktivierung der Prostataphosphatase wird durch Zusatz von $150 \mathrm{mmol} / \mathrm{l}$ 1-Pentanol zur Testlösung im Temperaturbereich von $20^{\circ} \mathrm{C}-40^{\circ} \mathrm{C}$ nicht beschleunigt.

Der Substratumsatz nimmt bei allen untersuchten Inkubationstemperaturen von $20^{\circ}-40^{\circ} \mathrm{C}$ linear zur Reaktionszeit zu (Abb. 8). Auch der Temperaturkoeffizient der 1-Pentanol-aktivierten Prostataphosphatase verändert sich nicht gegenüber dem alkoholfreien Testansatz. Erst bei Inkubationstemperaturen über $50^{\circ} \mathrm{C}$ beschleunigt 1-Pentanol die Hitzedenaturierung der Prostataphosphatase.

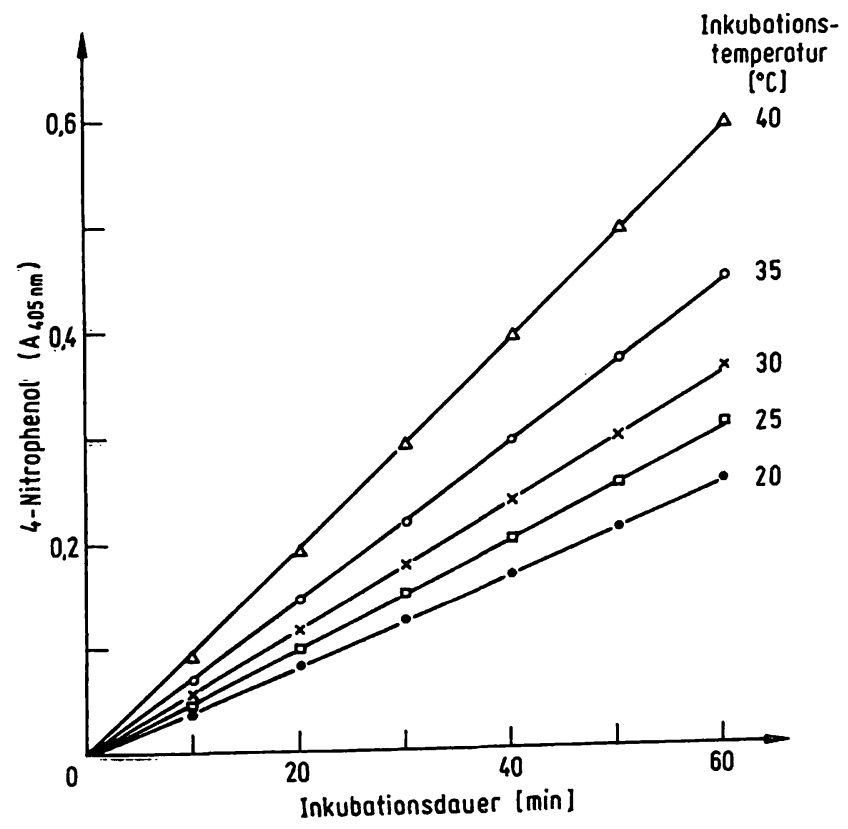

Abb. 8. Substratumsatz der Prostataphosphatase in Abhängigkeit zur Inkubationstemperatur und der Reaktionsdauer. Inkubation der Prostataphosphatase in $0,1 \mathrm{~mol} / 1 \mathrm{Natrium}$ citrat $\mathrm{pH} 5,25$ mit $5 \mathrm{mmol} / 1$ 4-Nitrophenylphosphat und $150 \mathrm{mmol} / 1 \mathrm{l}$-Pentanol bei den Temperaturen $20^{\circ} \mathrm{C}, 25^{\circ} \mathrm{C}$, $30^{\circ} \mathrm{C}, 35^{\circ} \mathrm{C}$ und $40^{\circ} \mathrm{C}$. Nach bestimmten Zeitintervallen wurde das freigesetzte 4-Nitrophenol bestimmt. 
Reagenzien zur Bestimmung der 1-Pentanolaktivierten Prostataphosphatase im Serum und Plasma

Im Serum und Plasma sind saure Phosphatasen aus verschiedenen Organen und Zelltypen vorhanden. Die Prostataphosphatase gilt als jener Teil der Gesamtaktivität der sauren Phosphatasen, der durch $L$-Tartrat gehemmt werden kann. Dementsprechend muß zur spezifischen Aktivitätsbestimmung der sauren Prostataphosphatase je ein Testansatz mit und ohne $L$-Tartrat durchgeführt werden.

Test zur Bestimmung der Gesamtaktivität der sauren Phosphatasen

$\mathrm{Zu}$

$0,5 \mathrm{ml}$ vorgewärmter Testlösung: $120 \mathrm{mmol} / 1 \mathrm{Natriumcitrat,}$ pH 5,25

$180 \mathrm{mmol} / \mathrm{l} 1$-Pentanol $6 \mathrm{mmol} / 1$ 4-Nitrophenylphosphat

werden

.

$0,1 \mathrm{ml}$ Serum oder Plasma zugemischt und während 30 Minuten bei $37^{\circ} \mathrm{C}$ inkubiert. Die enzymatische Reaktion wird durch Zugabe von

2,5 $\mathrm{ml} 1 \mathrm{~mol} / 1$ Natronlauge abgestoppt und das 4-Nitrophenolat bei der Wellenlänge $405 \mathrm{~nm}$ bestimmt.

Test zur Bestimmung der $L$-Tartrat-resistenten, sauren Phosphatasen

$\mathrm{Zu}$

$0,5 \mathrm{ml}$ vorgewärmter Testlösung: $120 \mathrm{mmol} / 1$ Natriumcitrat, $\mathrm{pH} 5,25$

$25 \mathrm{mmol} / 1 L$-Tartrat

$180 \mathrm{mmol} / 1$ 1-Pentanol

$6 \mathrm{mmol} / \mathrm{l}$ 4-Nitrophenylphos-

werden phat

$0,1 \mathrm{ml}$ Serum oder Plasma zugemischt und während 30 Minuten bei $37^{\circ} \mathrm{C}$ inkubiert. Die enzymatische Reaktion wird durch Zugabe von

2,5 $\mathrm{ml} 1 \mathrm{~mol} / 1$ Natronlauge abgestoppt und das 4-Nitrophenolat bei $405 \mathrm{~nm}$ bestimmt.

Zur Berechnung der Phosphatase-Aktivität (U/l) wird die gemessene Absorption $\left(\mathbf{A}_{405} \mathrm{~nm}\right)$ mit dem Faktor 55,85 multipliziert. Im Faktor 55,85 sind die Inkubationsdauer (1/30), die Relation Ansatzvolumen zu Probevolumen $(3,1 \mathrm{ml} / 0,1 \mathrm{ml})$ sowie der mikromolare $\mathrm{Ab}$ sorptionskoeffizient des 4-Nitrophenolates $\left(E_{405}: 18,5\right.$ $\mathrm{cm}^{2} / \mu \mathrm{mol}$ ) enthalten.

Eine vergleichende Aktivitätsbestimmung der sauren Phosphatasen in einigen Kontroll- und Patientenseren einerseits mit der konventionellen und andererseits mit der oben vorgeschlagenen Testmethode hat die $90 \%$ ige Aktivierung der sauren Prostataphosphatase durch Zusatz von $150 \mathrm{mmol} / 1$ 1-Pentanol bestätigt. Zudem hat sich bei diesem Versuch gezeigt, daß durch 1-Pentanol auch die „Nicht-Prostata“-Phosphatasen um 70\% ak tiviert werden. Einzig beim Kontrollserum „Precipath E“ konnte die saure Phosphatase durch 1-Pentanol nicht aktiviert werden.

\section{Diskussion}

Der Aktivierungsgrad der Prostataphosphatase scheint unter den gegebenen Testbedingungen von der Kettenlänge des eingesetzten Alkohols abhängig zu sein. Dabei wird mit dem 1-Pentanol die höchste Aktivierung von $90 \%$ erreicht. Bei noch höheren Alkoholen ist wegen der schlechten Löslichkeit die Alkoholkonzentration limitiert. Position șowie Anzahl der Hydroxylgruppen sind ebenfalls für die Enzymaktivierung von großer Bedeutung. Als optimal hat sich ein einwertiger primärer Alkohol (z. B. 1-Pentanol) erwiesen.

Appleyard (14) konnte durch Zusatz von 10\% Ethanol zur Testlösung die saure Prostataphosphatase um etwa $80 \%$ aktivieren. Als Substrat benutzte er das Phenolphthaleinphosphat. Mit den Substraten Phenylphosphat und 4-Nitrophenylphosphat konnten wir keinen so großen Aktivierungseffekt des Ethanols feststellen. Andererseits haben unsere Versuche mit dem Phenolphthaleinphosphat gezeigt, daß die Aktivitätsbestim- mung der sauren Phosphatasen mit diesem Substrat von äußerst komplexer Natur ist. Die diesbezüglichen Resultate werden an anderer Stelle mitgeteilt werden.

Substrat wie auch 1:Pentanol beeinflussen sich bezüglich ihrer Affinität zur Prostataphosphatase gegenseitig nicht. Hingegen wird die Reaktionsgeschwindigkeit durch 1-Pentanol erheblich beschleunigt. Auf Grund der dargelegten Resultate ist es naheliegend anzunehmen, daß die Prostataphosphatase neben der eigentlichen Phosphomonoesterase-Funktion auch noch die Fähigkeit besitzt, den Phosphatrest vom Substrat auf den Alkohol zu über-. tragen, was als Transphosphorylierung bezeichnet wird $(14,16)$. Diese Annahme wird durch die Beobachtung von Ostrowski (15) gestützt, der bei Anwesenheit von Ethanol in der Testlösung nach der ProstataphosphataseReaktion Ethylphosphat nachweisen konnte. Er fand auch, daß Ethylphosphat von der Prostataphosphatase äußerst langsam gespalten wird.

Die Transphosphorylierungsreaktion ist stark $\mathrm{pH}$-abhängig. Das pH-Optimum liegt im Bereich von $\mathrm{pH} 5,2$ bis 5,3 . Um diesen engen pH-Bereich einhaltẹn zu können, muß die Testlösung mit $100 \mathrm{mmol} / \mathrm{l}$ Natriumcitratpuffer versetzt werden; zur Kompensation der dadurch bedingten schlechteren Substrataffinität wird die Konzentration des 4-Nitrophenylphosphates auf $5 \mathrm{mmol} / \mathrm{l}$ heraufgesetzt.

Die Resultate der Tabelle 2 lassen vermuten, daß die Michaeliskonstante der Prostataphosphatase für 4-Nitrophenylphosphat in erster Linie von der Konzentration der Ionen und der ionisierten Gruppen abhängig ist. So ist zur Halbsättigung des Enzyms in Natriumcitratpuffer eine etwa dreifach höhere Substratkonzentration notwendig als in Natriumacetatpuffer gleichmolarer Konzentration.

Es überrascht, daß die saure Phosphatase im „Precipath

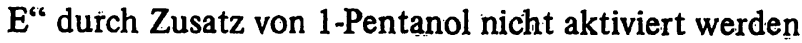


kann. Möglicherweise wurde diesem synthetischen Enzymkontrollserum auf Albuminbasis saure Phosphatase aus Kartoffeln zugesetzt, die auf Grund unserer Versuche durch 1-Pentanol nicht aktiviert werden kann.

Wir glauben mit dieser Arbeit einen Diskussionsbeitrag im Hinblick auf die Standardisierung der Bestimmungsmethode der Prostataphosphatase geleistet zu haben. Weitere Arbeiten werden erforderlich sein, um die vor- geschlagene Testmethode zu erproben und die von der Testmethode abhängigen Normalwerte der sauren Phosphatasen zu ermitteln.

\section{Danksagung}

Die Autoren danken Frau J. Marque und Frl. H. Dettmar für die effiziente technische Hilfe sowie Herrn Dr. K. Lauber, med.chem. Institut der Universität Bern für die Überlassung von Serumpools.

\section{Literatur}

1. Amador, E., Zimmermann, T. \& Wacker, W. (1963), J. Amer. Med. Ass. 185, 953-957.

2. Dayan, J. \& Wilson, I. (1964), Biochim. Biophys. Acta 81 , 620-623.

3. Wilson, I., Dayan, J. \& Cyr, K. (1964), J. Biol. Chem. 23.9, $4182-4185$.

4. Hausamen, T., Helger, R., Rick, W. \& Gross, W. (1967), Clin. Chim. Acta 15, 241-245.

5. Bowers, G., Kelley, J. \& Mc Comb, R. (1967), Clin. Chem. $13,608-610$.

6. Amador, E. (1972), Clin. Chem. 18, 94.

7. Mc Comb, R. \& Bowers, G. (1972), Clin. Chem. 18, 97-104.

8. Morin, L. (1973), Clin. Chem. 19, 1135-1138.
9. Neumann, H., Kezdy, F., Hsu, J. \& Rosenberg, I. (1975), Biochim. Biophys. Acta 39l, 292-300.

10. Deutsche Gesellschaft für Klinische Chemie (1972), diese Z. $10,182-192$.

11. Bessey, O., Lowry, O. \& Brock, M. (1946), J. Biol. Chem. $164,321-329$.

12. Bastiaanse, A. \& Meijers, C. (1968), diese Z. 6, 48-51.

13. Folin, O. \& Ciocalteu, V. (1927), J. Biol. Chem. 73, $627-650$.

14. Appleyard, J. (1948), Biochem. J. 42, 596-597.

15. Ostrowski, W. \& Barnard, E. (1973), Biochemistry 12, 3893-3898.

16. Jeffree, G. (1957), Biochim. Biophys. Acta 23, 155-166.

Dr. H. Gallati

Diagnostische Forschungsabteilung

F. Hoffmann-La Roche \& Co. AG, Grenzacherstraße 124

CH-4002 Basel

PD. Dr. M. Roth

Laboratoire Central

Hôpital Cantonal

CH-1211 Genève 4 

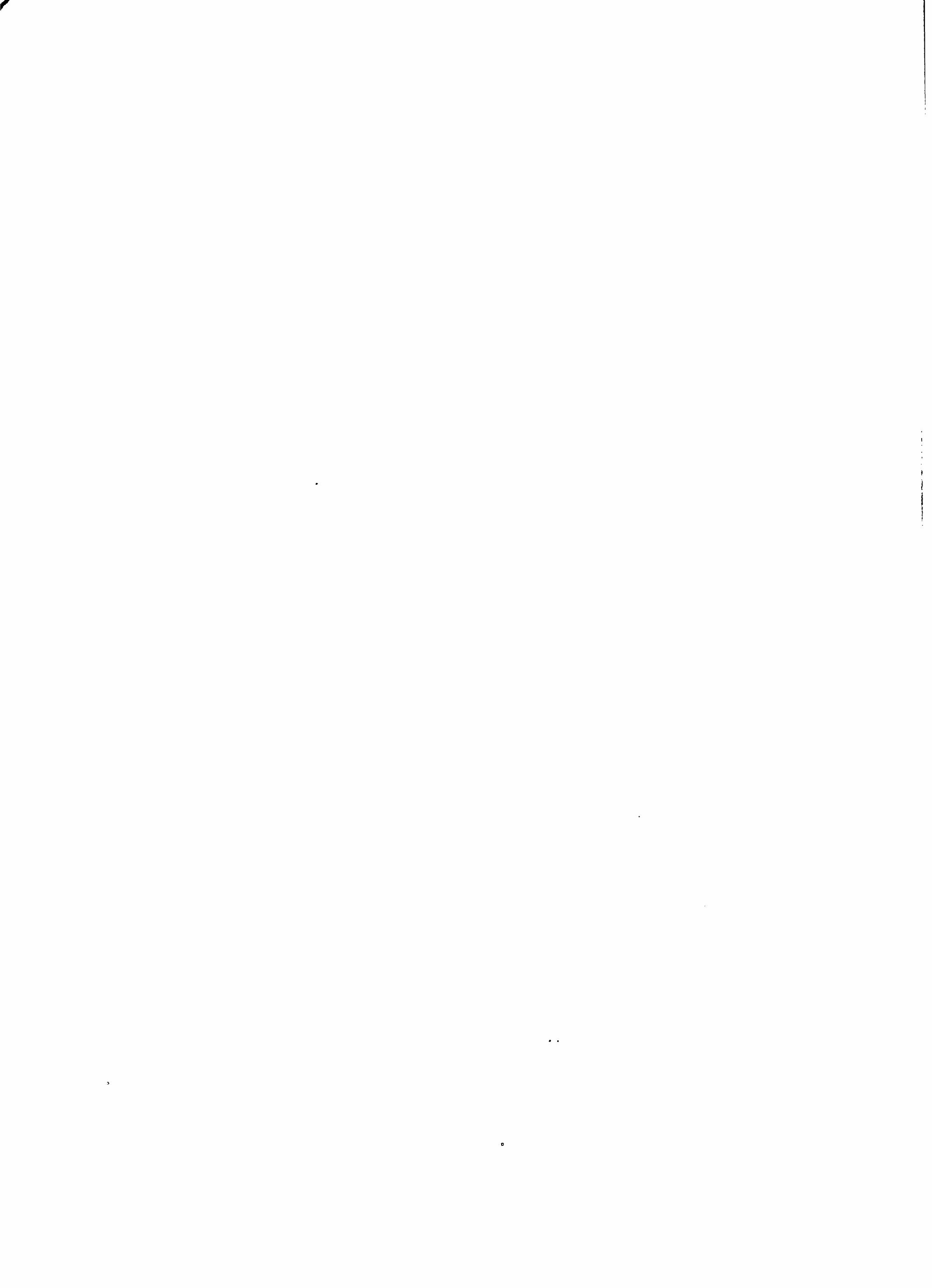